\section{Genomic characterisation of Staphylococcus aureus ST121 isolated from hospitalised patients in South Africa}

To the Editor: Staphylococcus aureus is a ubiquitous Gram-positive bacterium recognised as a major cause of minor to life-threatening infections in hospital and community settings. ${ }^{[1]}$ Its pathogenesis is associated with the expression of a variety of structural and secreted virulence factors that enable host colonisation, invasion of tissue and dissemination. Life-threatening staphylococcal infections are generally caused by the co-presence and combined actions of staphylococcal enterotoxins (sea-sej), toxic shock syndrome toxin-1 (tst-1), exfoliative toxins (eta and etb) and the pantonvalentine leucocidin. ${ }^{[2]}$ S. aureus is usually categorised as methicillinsusceptible S. aureus (MSSA) or methicillin-resistant S. aureus (MRSA) according to its susceptibility to beta-lactams. ${ }^{[3]}$ Although there is considerable controversy regarding the virulence of MSSA and MRSA isolates, it has been agreed that both have enormous virulence and pathogenicity capacities. ${ }^{[4]}$

The isolates described in this letter formed part of a bigger study investigating the molecular epidemiology of antibiotic resistance in clinical and carriage samples from patients admitted to public hospitals in uMgungundlovu District, KwaZulu-Natal Province, South Africa (SA). The isolates were identified using biochemical tests and antimicrobial susceptibility testing was performed by broth microdilution. The European Committee on Antimicrobial Susceptibility testing (EUCAST) breakpoints ${ }^{[5]}$ were used for interpretation of the results and S. aureus ATCC 29213 was used as the control. Whole-genome sequencing (WGS) analysis was performed on an Illumina MiSeq platform (Illumina Inc., USA) with $100 \times$ coverage. CLC Genomics Workbench version 10 (CLC Bio, QIAGEN, Denmark) and SPAdes ${ }^{[6]}$ were used for de novo assembly. The bacterial analysis pipeline of GoSeqIt (Denmark) tools was used to annotate and identify known acquired antibiotic-resistant genes via ResFinder, ${ }^{[7]}$ virulence factors using VirulenceFinder ${ }^{[8]}$ and mobile genetic elements through PlasmidFinder. ${ }^{[9]}$ Genomic DNA was extracted using the GenElute bacterial genomic DNA kit (SigmaAldrich, USA) according to the manufacturer's instructions. The multi-locus sequence type was determined from WGS data.

The two MSSAs, G703N1B1 (accession no. PGXA00000000) and G703N2B1 (accession no. PGWZ00000000) originated from nasal samples collected at admission and after 48 hours from a patient admitted to a tertiary hospital. Both isolates were ascribed to the sequence type 121 and exhibited multidrug resistance (Table 1). WGS confirmed this resistance profile by identifying several resistance genes in both isolates along with 18 virulence genes (Table 1).

To the best of our knowledge, this is the first report of communityacquired methicillin-susceptible $S$. aureus (CA-MSSA) ST121 isolated from a nasal carriage sample in SA. Rao et al.$^{[10]}$ demonstrated that the genetic lineage ST121 is an emerging and hypervirulent clone. Although we did not quantify the levels of production and mobilisation of the virulence determinants, the detection of 18 virulence genes in the CA-MSSA ST121 confirmed that this genetic lineage could probably contribute to severe outbreak situations, not only in communities but also in hospitals if infection control measures are not sufficiently implemented. This necessitates improvements in routine screening and reinforcement of infection, prevention and control measures.

Author contributions. RCF co-conceptualised the study, undertook sample collection and microbiological laboratory and data analyses, prepared tables, interpreted results, contributed to bioinformatics analysis, and drafted the manuscript. LLF undertook sample collection and microbiological laboratory analyses, contributed to bioinformatics analysis and vetted the results. MA undertook bioinformatics analysis. AI performed whole-genome sequencing analysis. SYE co-conceptualised the study and undertook critical revision of the manuscript. All authors read and approved the final manuscript.

Funding. This work was supported by the Antimicrobial Research Unit and College of Health Sciences of the University of KwaZulu-Natal. The National Research Foundation (NRF) funded this study through the NRF Incentive Funding for Rated Researchers (grant no. 85595), the NRF Competitive Grant for Rated Researchers (grant no. 106063) and the DST/NRF South African Research Chair in Antibiotic Resistance and One Health (grant no. 98342) awarded to SYE. The South African Medical Research Council also funded the WGS aspect of study through the Self-Initiated Research Grant. Any opinions, findings and conclusions or recommendations expressed in this letter are those of the authors and do not necessarily reflect the views of the organisations or agencies that provided support for the project. The funders had no role in the study design or the decision to submit the work for publication.

Conflicts of interest. SYE is a member of the Global Respiratory Infection Partnership sponsored by an unrestricted educational grant from Reckitt Benckiser, UK.

Table 1. Demographic and genotypic data of the methicillin-susceptible Staphylococcus aureus isolates

\begin{tabular}{|c|c|c|c|c|c|c|c|c|}
\hline \multirow[b]{2}{*}{ Isolate name } & \multicolumn{2}{|c|}{ Patient's details } & \multirow[b]{2}{*}{ Hospital } & \multirow[b]{2}{*}{ Timepoints } & \multirow[b]{2}{*}{ Resistance genes } & \multirow[b]{2}{*}{ Virulence factors } & \multirow[b]{2}{*}{ Plasmids } & \multirow[b]{2}{*}{ MLST } \\
\hline & Gender & Age (years) & & & & & & \\
\hline G703N1B1C1 & $\mathrm{F}$ & 37 & Tertiary & Admission & $\begin{array}{l}\text { blaZ, NOR(A), fosD, } \\
\text { dfrG, parC, parE, } \\
\text { gyrA, gyrB, fosB, } \\
\text { TcaA, TCaB, MATE, } \\
\text { AcrB }\end{array}$ & $\begin{array}{l}\text { aur, hlb, hlgA, hlgB, } \\
\text { hlgC, lukD, lukE, } \\
\text { sak, scn, seg, sei, } \\
\text { sem, sen, seo, seu, } \\
\text { splA, splB, eta }\end{array}$ & - & ST121 \\
\hline G703N2B1C1 & & & & After 48 hours & $\begin{array}{l}\text { blaZ, NOR(A), fosD, } \\
\text { dfrG, parC, parE, } \\
\text { gyrA, } \operatorname{gyr} B, \text { fos } B, \\
\text { TcaA }, \text { TCaB, MATE, } \\
\text { AcrB, } \operatorname{tet}(K)\end{array}$ & $\begin{array}{l}\text { aur, hlb, hlgA, hlgB, } \\
\text { hlgC, lukD, lukE, } \\
\text { sak, scn, seg, sei, } \\
\text { sem, sen, seo, seu, } \\
\text { splA, splB, eta }\end{array}$ & $\begin{array}{l}\text { repL } \\
\text { (pDLK1), } \\
\text { rep } \\
\text { (SAP060B), } \\
\text { rep } \\
\text { (SAP015B) }\end{array}$ & ST121 \\
\hline
\end{tabular}




\section{Raspail Carrel Founou}

Antimicrobial Research Unit, College of Health Sciences, University of KwaZulu-Natal, Durban, South Africa; and Department of Clinical Microbiology, Centre of Expertise and Biological Diagnostic of Cameroon, Yaoundé, Cameroon

czangue@yahoo.fr

\section{Luria Leslie Founou}

Antimicrobial Research Unit, College of Health Sciences, University of KwaZulu-Natal, Durban, South Africa; and Department of Food Safety and Environmental Microbiology, Centre of Expertise and Biological Diagnostic of Cameroon, Yaoundé, Cameroon

\section{Mushal Allam, Arshad Ismail}

Sequencing Core Facility, National Health Laboratory Service, Johannesburg, South Africa

\section{Sabiha Yusuf Essack}

Antimicrobial Research Unit, College of Health Sciences, University of KwaZulu-Natal, Durban, South Africa
1. Njoungang LL, Nwobegahay JM, Ayangma CR, et al. Prevalence and antibiotic resistance patterns of strains of Staphylococcus aureus isolated at the Yaounde Military Hospital, Cameroon. Microbiol Res Int 2015;3(4):56-63

2. Liu C, Chen Z-j, Sun Z, et al. Molecular characteristics and virulence factors in methicillin-susceptible, resistant, and heterogeneous vancomycin-intermediate Staphylococcus aureus from central-southern China. J Microbiol Immunol Infect 2015;48(5):490-496. https://doi.org/10.1016/j.jmii.2014.03.003

3. Shukla SK, Karow ME, Brady JM, et al. Virulence genes and genotypic associations in nasal carriage, community-associated methicillin-susceptible and methicillin-resistant USA400 Staphyloco
aureus isolates. J Clin Microbiol 2010;48(10):3582-3592. https://doi.org/10.1128/JCM.00657-10 aureus isolates. J Clin Microbiol 2010;48(10):3582-3592. https://doi.org/10.1128/JCM.00657-10
Jiménez JN, Ocampo AM, Vanegas JM, et al. Characterisation of virulence genes in methicillin susceptible and resistant Staphylococcus aureus isolates from a paediatric population in a university hospital of Medellín, Colombia. Mem Instit Oswaldo Cruz 2011;106(8):980-985.

5. European Committee on Antimicrobial Susceptibility Testing (EUCAST). Breakpoint Tables for Interpretation of MICs and Zone Diameters. Version 6.0, 2016

6. Bankevich A, Nurk S, Antipov D, et al. SPAdes: A new genome assembly algorithm and its applications to single-cell sequencing. J Comput Biol 2012;19(5):455-477 https://doi.org/10.1089/cmb.2012.0021 7. Zankari E, Hasman $\mathrm{H}$, Cosentino $\mathrm{S}$, et al. Identification of acquired antimicrobial resistance genes. J Antimicrob Chemother 2012;67(11):2640-2644. https://doi.org/10.1093/jac/dks261

8. Joensen KG, Scheutz F, Lund O, et al. Real-time whole-genome sequencing for routine typing, soensen KG, Scheutz $\mathrm{F}$, Lund $\mathrm{O}$, et al. Real-time whole-genome sequencing for routine typing,
surveillance, and outbreak detection of verotoxigenic Escherichia coli. J Clin Microbiol 2014;52(5):15011510. https://doi.org/10.1128/JCM.03617-13

9. Carattoli A, Zankari E, García-Fernández A, et al. In silico detection and typing of plasmids using PlasmidFinder and plasmid multilocus sequence typing. Antimicrob Agents Chemother 2014;58(7):3895-3903. https://doi.org/10.1128/AAC.02412-14

10. Rao Q, Shang W, Hu X, Rao X. Staphylococcus aureus ST121: A globally disseminated hypervirulent clone. J Med Microbiol 2015;64(12):1462-1473. https://doi.org/10.1099/jmm.0.000185

S Afr Med J 2018;108(12):1007-1008. DOI:10.7196/SAMJ.2018.v108i12.13713 